\title{
Epidemiological analysis of infertility in China
}

\author{
Liya $\mathrm{Ma}^{1, \mathrm{a}}$, Zhenghui Chang ${ }^{1, \mathrm{~b}}$ \\ ${ }^{1}$ Henan University of Traditional Chinese Medicine, Zhengzhou, Henan, 450046 \\ ${ }^{\mathrm{a}}$ email, ${ }^{\mathrm{b}}$ email
}

Keywords: Epidemiological Analysis, Infertility, Chinese Status

\begin{abstract}
Infertility is a very complex physiological process, is a special reproductive health defects, it is different from other clinical diseases, due to its physiological and psychological factors coexist, so it often has serious impact on the family, society. Most of the infertility patients are eager to get medical help, as soon as possible to get rid of the troubles of infertility. Therefore, it is necessary to study the causes of infertility and its influencing factors and diagnosis and treatment so as to provide basis for improving the population quality of our country.
\end{abstract}

\section{Introduction}

According to the provisions of the World Health Organization, both men and women if there is no desire not to give birth, cohabitation for more than a year, a normal sexual life and no contraceptive measures, still unable to conceive infertility. In 1975, WHO has been provided for 2 years, 1994 to 1 year. At present, Chinese scholars advocate to 2 years as the standard.

\section{Classification of Infertility}

Primary refers to the female sexual maturity has never been pregnant or never gave birth, secondary refers to the past there have been pregnancy (including full-term pregnancy, premature birth, abortion and ectopic pregnancy, hydatidiform mole, etc.) and again infertility. Male primary infertility is the man has never made a woman pregnant, male secondary infertility is the man had a woman to conceive, after infertility.

The former refers to the couple have congenital or acquired anatomical or functional defects, can not be corrected and can not conceive; the latter refers to the couple side due to some factors affect the pregnancy, after appropriate treatment may be pregnant.

Male and female, according to infertility factors in the man or woman and distinguish.

The former refers to the genital anatomical changes caused by infertility, which mainly refers to endocrine abnormalities caused by infertility.

Prevalence (prevalence) refers to a time point or time zone, a group of infertility in the percentage of infertility, reflecting the population under the static data. This probability can be obtained by census or random sampling. The prevalence of infertility (P)

$\mathrm{P}=$ number of people with infertility at a certain time (or time zone) (\%) / total number of people who were investigated for infertility in a certain region

Incidence rate (incidence rate) refers to the unit population in the unit time of infertility incidence (annual occurrence of new cases). As the dynamic data and is available through the anti-anti-study. As can not really determine the incidence of infertility time, the incidence of infertility is often difficult to accurately calculate. Therefore, the results of the epidemiological survey of infertility are often the prevalence of infertility.

\section{Factors Affecting the Prevalence of Infertility}

The incidence and prevalence of infertility affected by a variety of factors, in different countries and regions are very different, in 2001 the United States Prosser infertility causes attributed to biological factors, environmental factors and social factors one aspect. In today's world, the level of medical services has been increasing, but the incidence of infertility has increased significantly, and these 
three aspects of change has a direct relationship.

The factors of female infertility in biological factors are summarized as organic disease, endocrine factors, immune factors and mental factors. Male infertility causes hypothalamic pituitary lesions, testicular lesions, vas deferens and accessory glands, sperm transport dysfunction. The most common causes of infertility are ovulation, tubal disease, endometriosis, and semen problems. In recent years, sexually transmitted diseases (STD) is the main cause of infertility, Chlamydia trachomatis (CT) and AIDS is the leading cause of infertility in recent years, sexually transmitted diseases. CT can cause men and women genitourinary tract infection. WHO reports about 500 million people worldwide each year new sexually transmitted diseases, of which $18 \%$ is caused by the CT infection, and more associated with Neisseria gonorrhoeae. From 1994 to 2000, the incidence of women increased from $1.8 \%$ to $3.5 \%$, while men increased from $2.1 \%$ to $6.6 \%$, and the resulting fallopian tube infertility (TFI) accounted for 36\% of total TFI. In 2001 Gdoura reported $35.9 \%$ of Chlamydia trachomatis infection in male infertility, and 38\% of Chlamydia trachomatis were detected in female partners. Chlamydia trachomatis caused serious deterioration of semen quality. AIDS is the leading cause of infertility in Africa, Africa, the main reproductive behavior of the current performance of sexually transmitted diseases, AIDS and adolescent pregnancy. 2000 In Ulla, Africa Sahara Desert As a result of the spread of AIDS and sexually transmitted diseases, secondary infertility prevails in most African countries, with a prevalence rate ranging from $5 \%$ (Togo) to 23\% (middle) among people of childbearing age between 20 and 44 years of age Non republic), on the contrary, primary infertility is relatively low, only $1 / 3$ of the countries is greater than $3 \%$.

Environmental factors, people gradually realize that it occupies a pivotal position in infertility. The deterioration of the quality of the environment in which people have been living in recent years is silently affecting human fertility. Man-made chemicals are widely used, their types and production is increasing, people in the living, transportation, food, clothing, rest and other basic activities are constantly in contact with them, and in vivo accumulation. The impact of environmental pollution on female fertility mainly as irregular menstruation and ovulation disorder patients increased significantly, amenorrhea, oligomenorrhea and even premature ovarian failure are more common. Environmental pollution to men is mainly the impact of sperm quality, in 2001, France Oliva reported deteriorating environmental pollution caused by the deterioration of sperm quality caused by male infertility. Petrelli, Italy, 2002] reported that many environmental agents and insecticides exert a cytotoxic effect on human germ cells. Special insecticides destroy the endocrine system and damage male fertility.

\section{Chinese Current Infertility Epidemiological Discussion}

The survey of 2867 couples, the average age of the woman 26.139-31.19, minimum 15 years old, maximum 48 years old. The average age of the male 29.101- 41.13 years old, minimum 18 years old, maximum 52 years old. 9711\% of couples are first marriages. Education: Female primary school and below accounted for $217 \%$, junior high school accounted for $6414 \%$, high school and above accounted for 3219\%; male primary school and below accounted for 312\%, junior high school accounted for 5415\%, high school and above accounted for 4213\%. 2867 couples have 2396 couples in the non-contraceptive cohabitation after 1 year of pregnancy or contraceptive measures taken without pregnancy, 471 couples without contraceptive cohabitation 1 year period of infertility, infertility incidence of $16.14 \%$.

(1) Age: The incidence of infertility in women aged 35 years or older was significantly higher than that in other age groups $(\mathrm{P}=0.000)$. The incidence of infertility was significantly different among all age groups.

(2) educational level: couples for junior high school education more than half of the subjects, primary and below the group, junior high school and high school and above the incidence of infertility was no statistically significant difference, $P$ values were 0.1166 (woman) and 0.1322 ).

(3) Occupation: $42 \%$ for the farmers, $17 \%$ for workers or migrant workers, $41 \%$ for other occupations. According to the husband or wife of the occupational distribution of the three groups, 
the incidence of infertility were not statistically significant, p values were 0.1215 (woman) and 01910 (the man). (4) The woman's menstrual history: In this survey, women of childbearing age menarche average age of $14.170 \sim 11.21$ years old, menstrual cycle of the object accounted for 92.18\%, moderate menstrual accounted for $82.11 \%$. The incidence of infertility in normal and abnormal menstrual cycles was significantly different $(\mathrm{P}=0.1000)$. The difference in the incidence of infertility between the middle-term menstrual group and the more or less groups was statistically significant $(\mathrm{P}=0.1000)$. There was no significant correlation between dysmenorrhea and the incidence of infertility.

Logistic stepwise regression analysis was carried out using variables such as whether pregnancy was the dependent variable or not, and the variables of the biological characteristics of the men and women were used as independent variables. The results are shown in Table 2. Control of other factors, the incidence of infertility and the woman's age was positively related to the age of the infertility rate with the woman's age increased $(\mathrm{P}=01029$, $\mathrm{OR}=11049)$; men's age and infertility incidence was not significantly; The odds ratio of male infertility was significantly higher than that of normal menstrual cycle $(\mathrm{P}=01000$, $\mathrm{OR}=21365)$. There was no statistical significance in the multivariate analysis of male and female education.

The incidence of infertility by a variety of factors, in different countries, regions are very different, 2001 Prosser infertility causes attributed to biological factors, environmental factors and social factors in three aspects. In this study, we investigated the effects of social factors, including social demographic factors and social behavior factors, on the incidence of infertility in the two years after marriage. Socio-demographic factors: Research data confirm that as women age, fertility tends to decline, especially after the age of 35 is more obvious. The results of this study found that both men and women age of the couple and the incidence of infertility was significantly correlated, especially the woman's age. 35 years of age and above the incidence of infertility significantly increased the incidence of the group under the age of 3 times 3 times. The incidence of infertility in all age groups was also significantly different $(p>0105)$, and the results of multivariate analysis showed that the incidence of infertility was not statistically significant There was no significant correlation between the age of the man and the incidence of infertility. Social Behavioral Factors: Greb et al. (2005) showed that prolonged menstrual cycles lead to decreased fertility. This study found that menstrual cycle is normal or not, the amount of menstrual flow and infertility was significantly related. Menstrual cycle is normal, moderate menstrual women with low incidence of infertility. In the late eighties of last century, the survey of newly married couples showed that the education level was related to the incidence of infertility, but this study showed that the educational level of the couple was not related to the occurrence of infertility, which may not be as good as the overall education level , Junior college and above the proportion of low staff $(71.6 \%$ of the woman, the man $91.8 \%$ related.

Social and psychological factors also have an important impact on infertility. It includes the accelerated pace of life, lifestyle and public health main resources and conditions, directly or indirectly on the human endocrine system and fertility. Chinese reform and opening up policy, people's ideas have undergone great changes, many women postpone marriage age and childbearing age. The average age of infertile patients with an upward trend in the incidence of infertility also increased, many women over 35 years to come to the doctor. The concept of reproductive health refers to the physical, mental and social well-being, not just the absence of disease or weakness. Specific meaning includes human health and harmonious sexual psychology, normal sexual life, do not have to worry about sexually transmitted diseases and unplanned pregnancy; human fertility, but can scientifically regulate and control their fertility, freedom to decide whether or not Childbearing, childbirth, childbirth, childbirth and fertility; and that men and women have the right to be informed and to choose safe, effective, affordable and acceptable methods of fertility regulation, adequate health services, safe and smooth pregnancy, childbirth Healthy infants the best chance. Infertility and reproductive health: the literature reported that factors affecting reproductive health are: uncontrolled fertility and unwilling fertility; early marriage, early pregnancy, early childbearing; induced abortion; obstetric hemorrhage, dystocia, sepsis, child pain and unsafe abortion concurrent 
Reproductive and reproductive health, reproductive and reproductive health, reproductive health and reproductive health, reproductive and reproductive health, reproductive health and reproductive health, reproductive health and reproductive health, reproductive health and reproductive health.

\section{Conclusion}

Through the epidemiological survey of infertility in couples of childbearing age in this region, it was found that the incidence of female patients was higher than that of males. The proportion of urban secondary infertility was higher than that of rural secondary infertility and other characteristics, which is consistent with previous studies. Analysis of the causes and found that reproductive tract disease, systemic diseases, dirty sex life history is a common cause of infertility. Relative infertility patients is the main target of prevention and treatment, to strengthen the health of these couples of childbearing age publicity, change some bad habits, to prevent adverse sexual life history, timely detection of reproductive system diseases and reproductive function of the disease, so that they can get treatment in time.

\section{References}

[1] Wang Yannan, Wen Ji. 358 cases of infertility psychological factors in the investigation and research [J]. Progress in modern obstetrics and gynecology, 2015 (11)

[2] Shi Xin, Liu Lin, Zhang Xuehong. Advances in the study of assisted reproduction in monozygotic twins [J]. Progress in Modern Obstetrics and Gynecology, 2015 (06)

[3] Liu Jie, Zhong Fajiang, Li Liguo. Analysis of semen test results of male infertility patients with different syndromes [J]. Journal of Health Sciences, 2005 (07)

[4] Wang Yanhua.Chinese medicine sequential therapy on polycystic ovary syndrome in patients with sex hormones, insulin resistance levels and blood lipids [J]. Hebei Traditional Chinese Medicine, 2016 (06)

[5] Zhang Xinli, Li Yujie. Endometriosis r-AFS staging of infertility [J]. China Continuing Medical Education, 2008 (06) 